\title{
TRANSIT TIME OF ELECTRON TUNNELING THROUGH A POTENTIAL BARRIER
}

\author{
T. FIGIELSKI \\ Institute of Physics, Polish Academy of Sciences \\ Al. Lotników 32/46, 02-668 Warszawa, Poland
}

The average transit time of electron tunneling through a potential barrier is newly defined and examined without using the concept of a wave packet and without solving explicitly the time dependent Schrödinger equation.

PACS numbers: 03.65.-w, 73.40.Gk

The question: how much time it takes for an electron to tunnel through a barrier, is a basic problem in quantum mechanics, being today also of technological importance. Since 1932 [1] many different expressions for the tunneling time have been derived [2]. Because time has not a status of a dynamical variable in quantum. mechanics, and there is no operator for time, the definition of the tunneling time is not unequivocal.

Usually one defines the tunneling time by following the peak of a wave packet transmitted through the barrier region [3]. However, such a definition leads to a physically unacceptable result: when the barrier width tends to infinity, an average speed of tunneling electron tends also to infinity. This is likely a manifestation of the fact that the transmitted packet cannot represent the tunneling electron by itself [4].

We proposed a novel definition of the tunneling time that does not use the concept of a wave packet [5]. In this short note the definition has to be, of necessity, introduced in a heuristic way and the aim of this note may only be to show that in the simplest case it leads to intuitively acceptable consequences. It should be emphasized, however, that both the foundation of this approach and its consequences are by no means so trivial as it might appear from this presentation. Instead, they may be helpful to understand how to picture electrons when they are tunneling through a barrier.

Let us take into account a monoenergetic beam of electrons, having an energy $E$ and a wave number $k$, tunneling through a rectangular potential barrier. Suppose that at some moment of time the barrier height, $V_{0}$, is instantaneously changed by $\Delta V$. If a change in the probability current from $j$ to $j+\delta j$ at the front wall of the barrier is followed by the same change at the back wall, but occurring at a time interval $\tau$ later, it means that a current carrier had to spend the time $\tau$ on traveling through the barrier region, and thus this $\tau$ has a meaning of the transit 

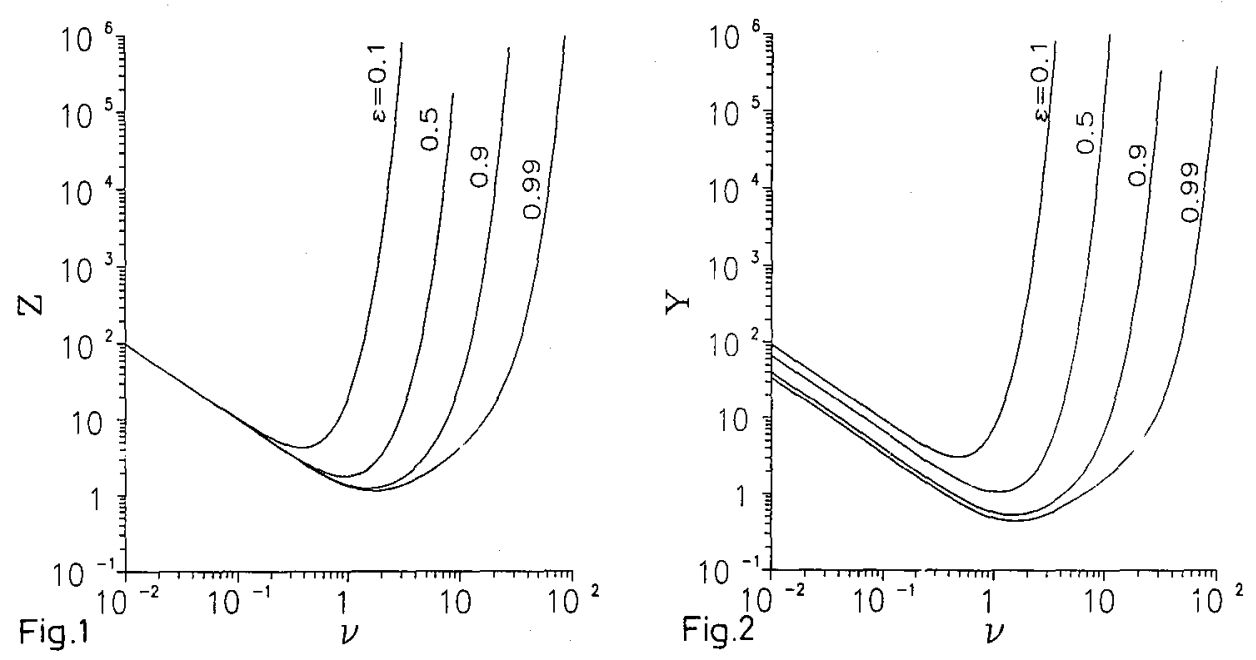

Fig. 1. Universal set of curves for determining the average steady-state tunneling time $\langle\tau(\infty)\rangle$. The time is given by Eq. (3), where $Z(\nu, \varepsilon)$ curves are displayed in the figure as a function of $\nu=w k$, for different values of the parameter $\varepsilon=E / V_{0}$.

Fig. 2. The same as in Fig. 1 but for the momentary tunneling time $\langle\tau(0)\rangle . Y(\nu, \varepsilon)$ curves are displayed in the figure.

time. The complete transient process involves, in general, an infinite assemblage of such times that form a distribution. An average value taken from this distribution represents a mean tunneling time relevant to a considered process.

One can directly write an expression for this average time invoking the continuity equation for the probability

$$
\frac{\partial \rho(x, t)}{\partial t}=-\frac{\partial j(x, t)}{\partial x}
$$

where $\rho(x, t)=|\Psi(x, t)|^{2}$ is the probability density. After integrating Eq. (1) over the barrier width, $w$, and over the time, one obtains a steady change in the total probability inside the barrier region: $\Delta Q \equiv \int_{0}^{w} \rho(x, 0) \mathrm{d} x-\int_{0}^{w} \rho(x, \infty) \mathrm{d} x$, caused by the change in the barrier height. If the corresponding change of the probability current through the barrier is $\Delta j$, then the average tunneling time can be written as

$$
\langle\tau(\Delta V)\rangle=\Delta Q / \Delta j
$$

For two limiting cases $\Delta V \rightarrow 0$ and $\Delta V \rightarrow \infty$, the expression (2) takes the following form: $\langle\tau(0)\rangle=(\partial Q / \partial V) /(\partial j / \partial V)$ and $\langle\tau(\infty)\rangle=Q / j$, respectively. The first time describes a response to infinitesimal change in the barrier height, while the second one is relevant to a steady state tunneling.

After introducing the dimensionless parameters $\varepsilon=E / V_{0}$ and $\nu=w k$, these times can be written as

$$
\langle\tau(0)\rangle=m w^{2} Y(\nu, \varepsilon) / \hbar, \quad\langle\tau(\infty)\rangle=m w^{2} Z(\nu, \varepsilon) / \hbar,
$$


where the functions $Y(\nu, \varepsilon)$ and $Z(\nu, \varepsilon)$ are displayed in Figs. 1 and 2.

There are two ranges of different behavior of the tunneling time for each $\varepsilon$, one corresponding to the negative and the other to the positive slope of $Y$ (or $Z$ ) versus $\nu$, which may be called the transparent barrier range and the opaque barrier range, respectively. Of course, the tunneling time is always an increasing function of the barrier width. In the transparent barrier limit the average steady-state tunneling time, $\langle\tau(\infty)\rangle$, is proportional to the barrier width, therefore, an electron traverses the barrier region with a constant average speed, equal to the speed it had before entering this region. In the opaque barrier limit, the tunneling time increases exponentially with the barrier width; thus, an average speed of tunneling electron is always finite.

It is worth noting that despite an overall similarity, the behavior of the time $\langle\tau(0)\rangle$ differs from that of $\langle\tau(\infty)\rangle$ in an essential point. Namely, in the first case an average speed of electron traversing the barrier exceeds the speed of the incident electron!

In conclusion, it has to be emphasized that the general expression (2) may be referred to other related problems as well, e.g. it describes exactly the period of single-electron-tunneling coherent oscillations that appear in small tunnel junctions at low temperature.

Thanks are due to Dr. E. Kaczmarek for her assistance. This work was supported by the Committee for Scientific Research grant No 204619101.

\section{References}

[1] L.A. MacColl, Phys. Rev. 40, 621 (1932).

[2] E.H. Hauge, J.A. Stovneng, Rev. Mod. Phys. 61, 917 (1989).

[3] T.E. Hartman, J. Appl. Phys. 33, 3427 (1962).

[4] K.W.H. Stevens, J. Phys. C, Solid State Phys. 17, 5735 (1984).

[5] T. Figielski, submitted to Phys. Rev. Lett. 